\title{
REVIEW
}

\section{Revisiting the Interaction Force Measurement between Lipid Bilayers Using a Surface Forces Apparatus (SFA)}

\author{
Dong Woog Lee
}

School of Energy and Chemical Engineering, Ulsan National Institute of Science and Technology, 50 UNIST-gil, Ulju-gun 44919, Republic of KOREA

\begin{abstract}
In this review, previous researches that measured intermembrane forces using the Surface Forces Apparatus are recapitulated. Different types of interaction forces are reported between two lipid bilayers including non-specific interactions (e.g., van der Waals, electrostatic, steric hydration, thermal undulation, and hydrophobic) and specific interactions (e.g., ligand-receptor). By measuring absolute distance and interaction forces at the sub-angstrom level and at a few nano-Newtons resolution, respectively, magnitudes, working ranges, and decay lengths of interaction between lipid bilayers are investigated. Utilizing recently developed fluorescence microscopy attachments, simultaneous fluorescence imaging of membrane proteins and lipid phases can be performed during approach/separation cycles of two lipid bilayer deposited surfaces, which can reveal cooperative effects between lipid phases and various types of membrane proteins.
\end{abstract}

Key words: surface forces apparatus, lipid bilayers, intermembrane forces, van der Waals forces, electrostatic forces, entropic forces, hydrophobic forces, membrane fusion, specific interaction

\section{Introduction - Surface Forces Apparatus (SFA)}

The Surface Forces Apparatuses (SFA) has been used for decades to measure interaction forces and absolute distance between two macroscopic surfaces. The first version of an SFA was developed by Tabor, Winterton and Israelachvili in the $1970 \mathrm{~s}^{1,2)}$, and it was gradually improved by Israelachvili and coworkers to measure various types of interaction forces such as van der Waals (vdW), electrostatic, hydrophobic/hydration, specific/non-specific adhesion, and polymer-mediated adhesive and steric forces. Currently, an SFA can measure distance up to a sub-angstrom with a force resolution of a few nano-Newtons ${ }^{3 \text { ) }}$. Along with improving resolution, various attachments constantly have been developed such as (i) a bimorph slider attachment that can actuate lateral sliding and measure friction forces ${ }^{3)}$, (ii) an optical and fluorescence attachment that enables in situ optical and fluorescence imaging ${ }^{4,5)}$, and (iii) an electrochemical cell that can apply and measure electric potential between two surfaces ${ }^{6,7)}$.
1.1 Absolute distance and interaction force measurements

The absolute distance between two opposing surfaces is measured by multiple beam interferometry ${ }^{8)}$, which also provides the quantitative shapes of the surfaces. Back-silvered (thickness $\sim 50 \mathrm{~nm}$ ) and freshly cleaved muscovite mica (thickness $\sim 2-5 \mu \mathrm{m}$ ) is generally used as substrate, which is molecularly smooth (RMS roughness $\sim 0.04 \mathrm{~nm}$ ). Two back-silvered mica substrates are glued onto two cylindrical-shaped sapphire disks and mounted in the SFA chamber in a cross-cylinder geometry, which is mathematically equivalent to a sphere on a flat geometry. When the white light passes through the mounted surfaces, the silver layers of opposing surfaces act as an optical trap, providing an interference pattern known as fringes at equal chromatic order (FECO). By analyzing the FECO, absolute distance between mica surfaces can be measured with the resolution of a sub-angstrom (Fig. 1).

The interaction forces between two opposing surfaces are measured by the deflection of the double cantilever spring with a spring constant of $k$, which is attached to the

\footnotetext{
*Correspondence to: Dong Woog Lee, School of Energy and Chemical Engineering, Ulsan National Institute of Science and Technology, 50 UNIST-gil, Ulju-gun 44919, Republic of KOREA E-mail: dongwoog.lee@ unist.ac.kr Accepted August 16, 2018 (received for review May 11, 2018) Journal of Oleo Science ISSN 1345-8957 print / ISSN 1347-3352 online
} http://www.jstage.jst.go.jp/browse/jos/ http://mc.manusriptcentral.com/jjocs This review is presented to the winner of the Young Fellow Award at 2nd Asian Conference on Oleo Science (ACOS 2017) and 56th Annual Meeting of Japan Oil Chemists' Society (JOCS). 

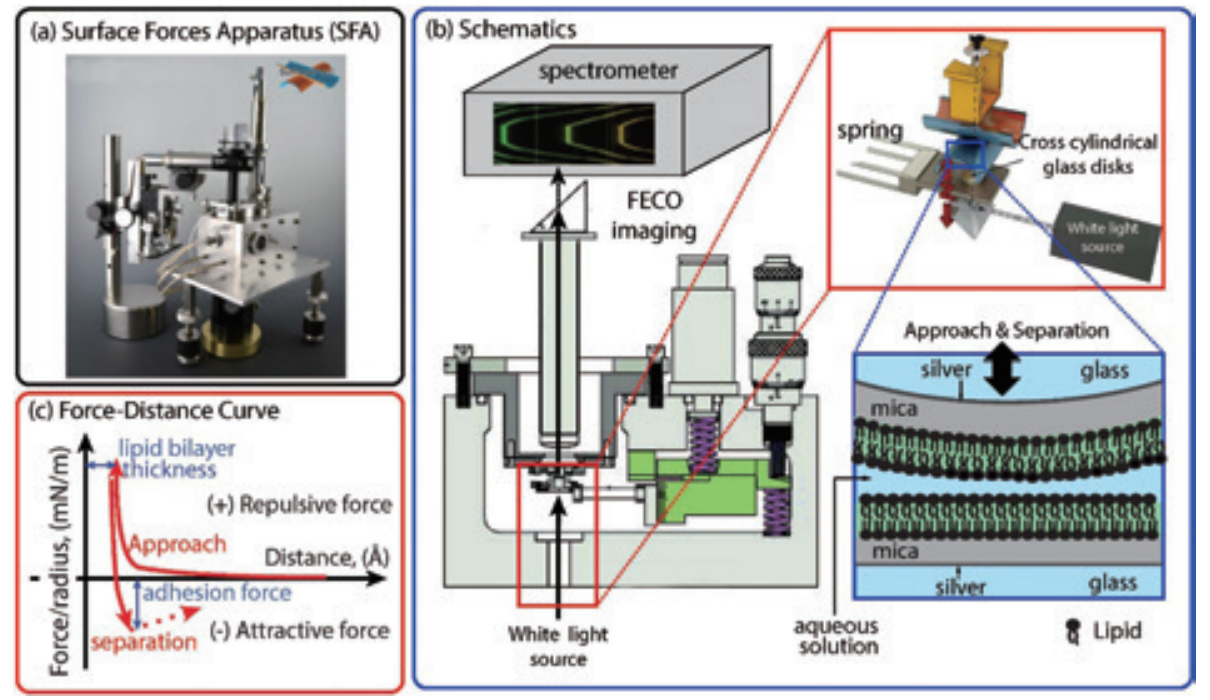

Fig. 1 Schematics of (a) a surface forces apparatus (SFA), (b) an SFA experiment set-up for measuring interaction forces between lipid bilayers, and (c) an example of the force-distance curve.

lower disk mount. The deflection of the spring $(\Delta D)$ is measured by analyzing the FECO, and by applying Hooke's law $(F=k \Delta D)$ the interaction forces could be calculated. Consequently, the force resolution is highly related to the spring constant and the distance resolution, which is currently about a few nano-Newtons.

Measured distance and interaction forces between two surfaces of interest are plotted to generate a force-distance $(F-D)$ curve to obtain various information, such as (steric) hard wall, adhesion force, and repulsive force (Fig. 1c). The $F-D$ curve can be fitted to various theories (e.g., Derjaguin-Landau-Verwey-Overbeek [DLVO], Alexander-de Gennes polymer theory)in order to investigate the types and magnitudes of engaging interaction forces.

\subsection{SFA experiment set-up for measuring interaction forces between lipid bilayers}

Recent technical improvements have advanced the field of membrane science ${ }^{9,10)}$, however, in terms of measuring intermembrane forces, atomic force microscopy (AFM) is one of the most used force spectroscopic techniques ${ }^{11)}$. AFM indeed is a powerful technique that can simultaneously image the morphology of a membrane and measure forces, however, it has several limitations. Due to the distance measuring methodology of AFM, only relative distance between lipid bilayers can be measured by setting steric hard wall as "zero" distance. Consequently, it is difficult to accurately measure the thickness of the lipid membranes, which is critical to decide the planes of origin of various interaction forces. Moreover, due to the rough nature of the probing tip, it is difficult to theoretically analyze the measured $F-D$ curve correctly. Thus, an SFA, which measures the absolute distance between molecularly smooth mica surfaces, can be very advantageous for inter- membrane force measurements.

Measuring interaction forces between lipid bilayers using an SFA is more subtle compared to other typical SFA experiments due to the high fragility of lipid bilayers when exposed to air or compression. Moreover, lipid molecules can slowly diffuse out to an aqueous environment, which can result in depletion of lipid molecules and form a thinner outer monolayer or even membrane holes. In order to overcome these complications of lipid bilayer SFA experiments, special tools and methods for bilayer preparation and transfer have been developed over the years.

In order to deposit lipid bilayers onto mica, two different methods are mainly used: (i) Langmuir-Blodgett (LB) deposition $^{12,13)}$ and (ii) spontaneous self-assembly ${ }^{14,15)}$. For LB deposition, a special type of disk holder (also known as a dove-tail disk holder) is used (Fig. 2a). Dove-tail disk holder can be easily held with a cross-type, self-locking tweezer that is punched with small screws at the tips (Fig. $2 \mathrm{~b}, 2 \mathrm{c}$ ). A dove-tail disk or disk holding tweezer is controlled by the dipper of the LB trough (Fig. 2d). After the deposition of lipid bilayers, dove-tail disks are kept inside small glass petri dishes prefilled with buffer solution to avoid air exposure (Fig. 2e). The disks kept in petri dishes are transferred into the SFA chamber, which is also prefilled with buffer solution. Using this transfer method, LBdeposited lipid bilayers are kept under the buffer at all times, which is necessary for the integrity of the bilayers' structures. Furthermore, buffer solution can be saturated with lipids to avoid slow diffusion of lipid molecules from bilayers to the bulk buffer solution. The advantages of LB deposition compared to the spontaneous self-assembly method are as follows: (i) asymmetric bilayers can be easily deposited, (ii) almost any type of lipid can be used while anionic lipids or surfactants cannot be used with the spon- 

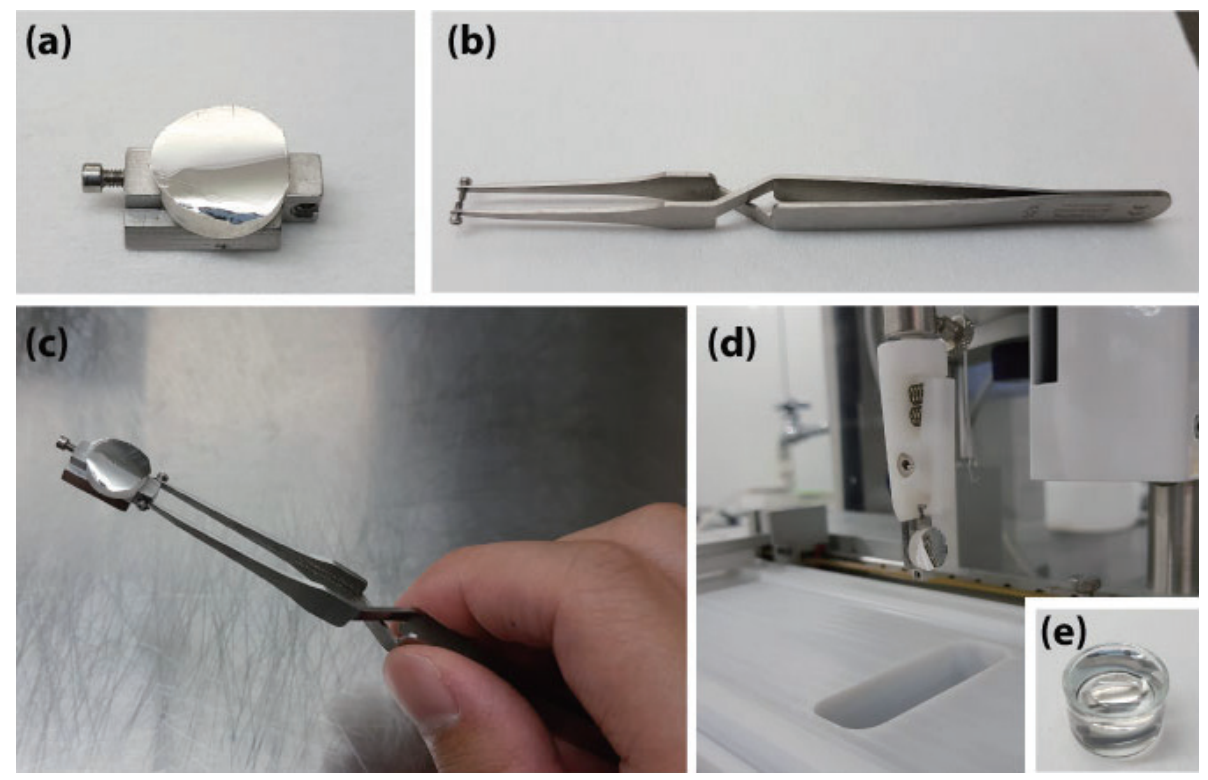

Fig. 2 Photographs of (a) a dovetail disk holder, (b)a cross tweezer for dovetail disk holder, (c) a cross tweezer holding the dovetail disk holder, (d) a dipper of Langmuir Blodgett(LB) trough holding the dovetail disk holder for lipid bilayer deposition, and (e) a small petri dish filled with buffer used for carrying lipid bilayer deposited disk without exposing lipid bilayer to air.

taneous self-assembly method, and(iii) the molecular area of lipid molecules can be controlled, which determines the "phase" of the lipid bilayers.

After mounting two lipid bilayers in a buffer-filled SFA chamber, molecules of interest can be injected to the chamber, which can mediate/tune the interaction forces between bilayers. These molecules include proteins, polysaccharides, polymers, ions, and so on. Also, during the second lipid layer deposition process, a desired quantity of molecule-conjugated lipids can be used, which makes lipid bilayers become covalently bound molecules on their headgroups. The surface density of the molecules can be controlled by simply tuning the ratio between the pristine lipids and molecule-conjugated lipids. Specific examples of these studies will be mentioned further in a later section of this review.

\section{Interaction forces between lipid bilayers}

Accurately measuring interaction forces and properly analyzing them could reveal the fundamental interactive forces during cell adhesion and membrane fusion, which has been of great interest since the 1980s. Early studies of interaction forces measurement between lipid bilayers using the $\mathrm{SFA}^{16-19)}$ identified that three types of forces dominate the full interbilayer force laws: (i) attractive van der Waals, (ii) repulsive electrostatic (double-layer), and (iii) short range repulsive hydration.

\subsection{The van der Waals (vdW) interaction}

The vdW interaction is a universal force, such as gravitational force, that acts between all atoms and molecules, even totally neutral molecules such as hydrocarbons. The $\mathrm{vdW}$ interaction arises from the fluctuation in the electric dipole moments of molecules, which varies with the inverse sixth power of the distance, $r$ :

$$
E(r)=-C_{\mathrm{vdw}} / r^{6}
$$

where $C_{\text {vdw }}$ is a constant that depends on the optical properties of interacting atoms. The vdW interaction between macroscopic bodies is longer ranged and generally described by the Hamaker constant, $A$. Specifically, for SFA experiments where two cross-cylindrical surfaces are typically used, vdW interaction energy follows following the relation:

$$
E(D)=-A R / D
$$

where $R$ is the radius of the cylinder and $D$ is the distance between the cylinders. The vdW interaction energy between a lipid bilayer and a liposome(flat-sphere) or between liposomes (sphere-sphere) also follows $1 / D$ dependence.

The Hamaker constant, $A$, of two bodies 1 and 2 interacting in a medium 3 can be expressed by the Lifshitzs theory ${ }^{20)}$ : 


$$
\begin{aligned}
A_{\text {tot }}= & A_{v=0}+A_{v>0} \\
& \approx \frac{3}{4} k T\left(\frac{\varepsilon_{1}-\varepsilon_{3}}{\varepsilon_{1}+\varepsilon_{3}}\right)\left(\frac{\varepsilon_{2}-\varepsilon_{3}}{\varepsilon_{2}+\varepsilon_{3}}\right) \\
& +\frac{3 h v_{e}}{8 \sqrt{2}} \frac{\left(n_{1}^{2}-n_{3}^{2}\right)\left(n_{2}^{2}-n_{3}^{2}\right)}{\left(n_{1}^{2}+n_{3}^{2}\right) \frac{1}{2}\left(n_{2}^{2}+n_{3}^{2}\right)^{\frac{1}{2}}\left\{\left(n_{1}^{2}-n_{3}^{2}\right)+\left(n_{2}^{2}-n_{3}^{2}\right)\right\}}
\end{aligned}
$$

where $v_{e}$ is the electronic absorption frequency, and $\varepsilon_{x}$ and $n_{x}$ are the dielectric constants and the refractive indices for media $x$, respectively.

Marra and Israelachvili ${ }^{17)}$ were able to measure the full force laws between various types of lipid bilayers. By fitting the $F-D$ curve to DLVO theory, which includes vdW and electrostatic double layer (DL) forces, they found that the Hamaker constant $(A)$ falls within the range $A=(7.5 \pm 1.5)$ $\times 10^{-21} \mathrm{~J}$, for supported lipid bilayers in water, which decreases to about $A \approx 5 \times 10^{-21} \mathrm{~J}$ in physiological salt concentrations at distances beyond Debye length, due to the electrostatic screening of the zero-frequency contribution by electrolyte ions. Also, at separation distances beyond 15 $\mathrm{nm}$, the dispersion contribution of the vdW force is known to be retarded, which makes the vdW force too weak to be important. Furthermore, the van der Waals plane of origin was found to be on average $\sim 0.5 \mathrm{~nm}$ away from the anhydrous headgroup configuration (Fig. 3 ).

\subsection{Electrostatic Double-Layer(DL) force}

The electrostatic DL force is strong, long ranged, and always exists between two macromolecules or surfaces in aqueous solutions as long as they are charged. In most cases, biological surfaces, molecules, and molecular groups are charged because they are inclined to be ionized in an aqueous environment. The electrostatic interaction energy between two similarly charged surfaces can be expressed by the following equation, which is an exponentially decaying function:

$$
E(D) \approx+C_{\mathrm{ES}} \mathrm{e}^{-\kappa D}
$$

where $C_{\mathrm{ES}}$ is a constant that depends on the surface charge density, solution conditions, and the geometry of the interacting surfaces. The constant $C_{\text {ES }}$ can be calculated by using the Poisson Boltzmann equation and/or other theories $^{21-27)}$. The Debye length $\left(\kappa^{-1}\right)$, which is the characteristic decay length of the interaction, only depends on the solution conditions:

$$
\kappa^{-1}=\left(\sum_{i} \frac{\rho_{i \infty} e^{2} z_{i}^{2}}{\varepsilon \varepsilon_{0} k T}\right)^{-1 / 2}
$$

where $\rho_{i \infty}$ is the number density of ions of valency $z_{\mathrm{i}}$ in the bulk solution, $\varepsilon$ is the dielectric constant of media, $\varepsilon_{0}$ is the vacuum permittivity, and $e$ is the electronic charge. In physiological conditions, Debye length is approximately 0.8 nm.

For SFA experiments that use two surfaces in cross-cylindrical geometry, electric DL interaction energy between two similar surfaces can be written as:

$$
E(D) \approx R Z e^{-\kappa D}
$$

where $R$ is the radius of the cylinders and $Z$ is a constant that can be expressed as functions of surface potential, $\psi_{0}$ :

$$
Z=64 \pi \varepsilon \varepsilon_{0}\left(\frac{k_{\mathrm{B}} T}{e}\right)^{2} \tanh ^{2}\left(z e \psi_{0} / 4 k_{B} T\right)
$$

Among the early works of measuring DLVO forces between lipid bilayers, Marra measured electrostatic DL forces between two charged lipid bilayers (dipalmitolphosphatidyl glycerol, $\mathrm{DPPG}^{-}$) in various salt types and concentrations $^{19)}$ (Fig. 4). This work showed that the interaction force law between lipid bilayers follows DLVO theory in buffer with monovalent counter-ions, whereas in buffer with divalent counter-ions induces higher adhesion at shorter ranges compared to the value predicted from vdW. This effect was attributed to the combination of "ion-cor-

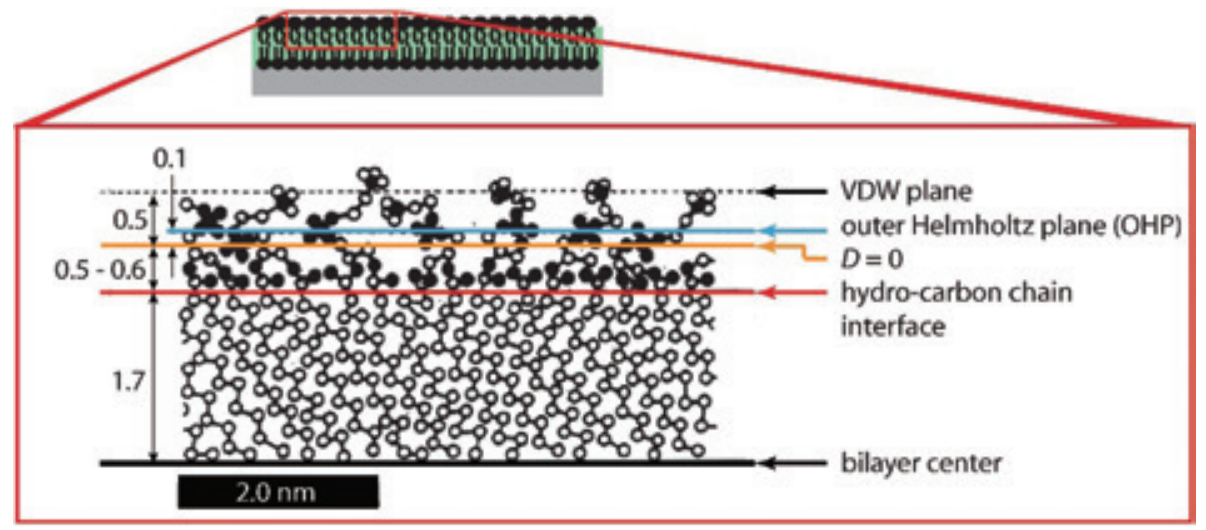

Fig. 3 Schematic of DPPC bilayer showing the position of outer Helmholtz plane, plane of origin of the van der Waals interaction, relative to anhydrous headgroup configuration $(D=0)$ which is a hypothetical "contact" between two lipid bilayers with no water left. [Modified from Marra and Israelachvili, $1985^{17)}$.] 


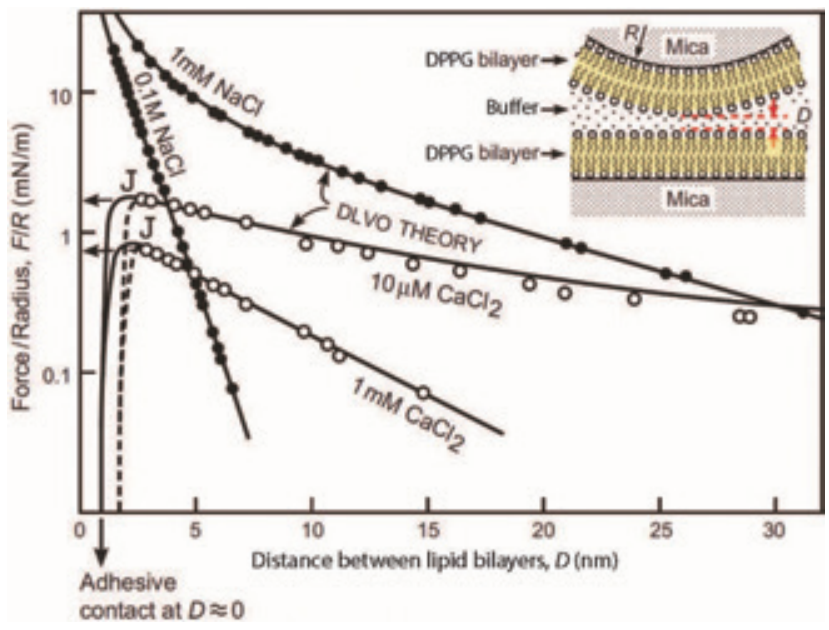

Fig. 4 Measured interaction forces between dipalmitolphosphatidyl glycerol (DPPG) bilayers. The solid lines are the predicted DLVO plot using a Hamaker constant of $A=6 \times 10^{-21} \mathrm{~J}$. In $\mathrm{NaCl}$ solution, DLVO fitting suggests two fully charged surfaces while in $\mathrm{CaCl}_{2}$ solutions, the surface charge is reduced due to ion binding. [Adapted and modified from Marra \& Israelachvili, $1985^{17}$; Marra, $1986^{19)}$; Israelachivili, $\left.2011^{20)}\right]$.

relation" or "charge fluctuation" ${ }^{28,29)}$ and "ion condensation" effects ${ }^{30)}$.

\subsection{Repulsive entropic forces}

2.3.1 Steric hydration force: Protrusion and headgroup overlap

First proposed by Langmuir ${ }^{31}$, steric hydration forces between lipid bilayers are strongly repulsive short-ranged (1-3 nm) forces that dominate over the vdW and electric DL forces at small separation distances. Due to their shortranged nature, which decays exponentially with a decay length $(\sim 0.2 \mathrm{~nm})$ close to the size of a water molecule, steric hydration forces were considered to have originated from water structuring. However, it was found that these forces become stronger as temperature increases, which is opposite from what is expected because the water structure is considered to be more disordered at higher temperatures. Further studies revealed that the steric hydration forces have entropic origin ${ }^{32-34)}$, which arises from surface groups overlapping in a liquid (Fig. 5).

Consequently, the steric hydration forces are determined by two factors in lipid bilayers: (i) the degree of lipid molecule protrusion from the lipid bilayer surfaces (i.e., the protrusion force) and (ii) the hydrated size of the lipid headgroups (i.e., the headgroup overlap force). These two forces are highly affected by the degrees of headgroup hydration and hydrophilicity of lipid/water interface. The hydration of the lipid headgroups can occur by their intrinsic hydrophilicity and/or induced hydrophilicity upon ion

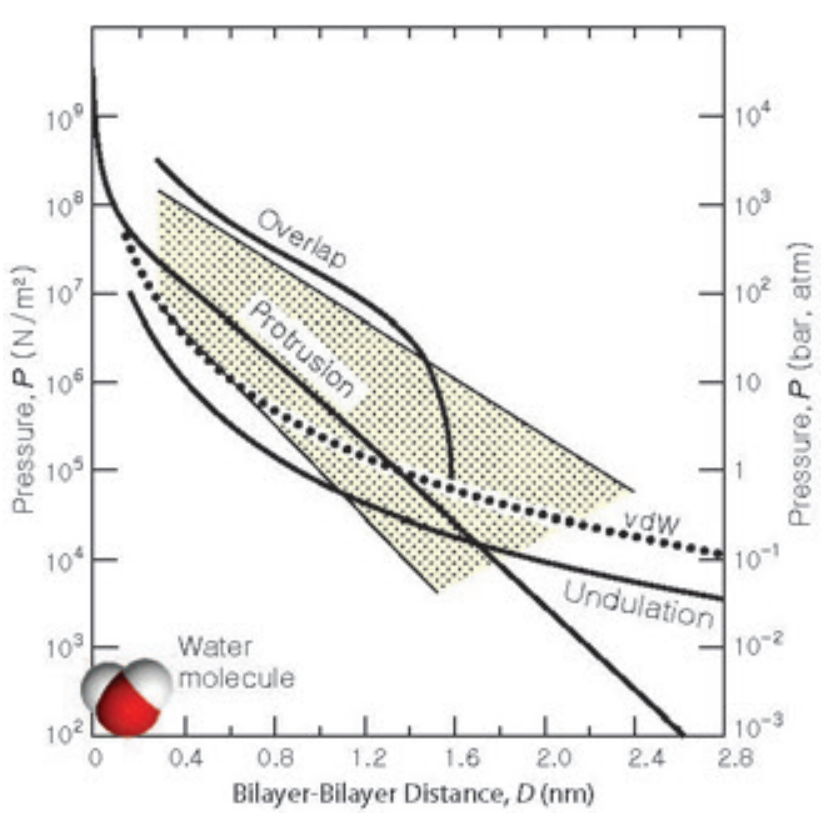

Fig. 5 Theoretical plots of different types of repulsive entropic forces (e.g., undulation, protrusion, and headgroup overlap) compared with vdW force. The shaded region indicates the limits of the repulsive forces between lecithin bilayers. [Adapted and modified from Israelachvili, $2011^{20)}$.]

binding, so called "charge regulation."

In more recent research on the hydration forces using an SFA, Schrader et al. ${ }^{35)}$ showed that the steric hydration forces between dipalmitoylphosphatidylcholine (DPPC) bilayers can be reduced by adding Dimethyl sulfoxide (DMSO), which not only decreases the hydrated volume of the lipid headgroups but also suppresses the headgroups' thermal motion by collapsing them onto the surface. This work fitted Alexander-de Gennes brush theory to the full $F-D$ curve by treating headgroups as brushes, and the results show that as the DMSO concentration increases the brush length decreases. Above 10\% DMSO concentration, brush theory could not be applied, and this is an indication of headgroup collapse (Fig. 6) .

\subsubsection{Undulation forces}

The long-range undulation force arises from long wavelength thermal ripples of lipid bilayers. When the separation distance between two lipid bilayers becomes small (even smaller than the amplitude of the thermal ripples), the lipid membranes become "confined," which reduces the entropy of the system, resulting in a repulsive force. The undulation force is mainly dominated by the bending modulus of the lipid bilayers, which is mostly affected by the phase of the lipid bilayers. The phase of the lipid bilayers depends on the length of hydrocarbons, degree of saturation, headgroup types, and undoubtedly the temperature. Helfrich and coworkers ${ }^{36,37)}$ showed that the undulation force can be expressed by the following equation: 

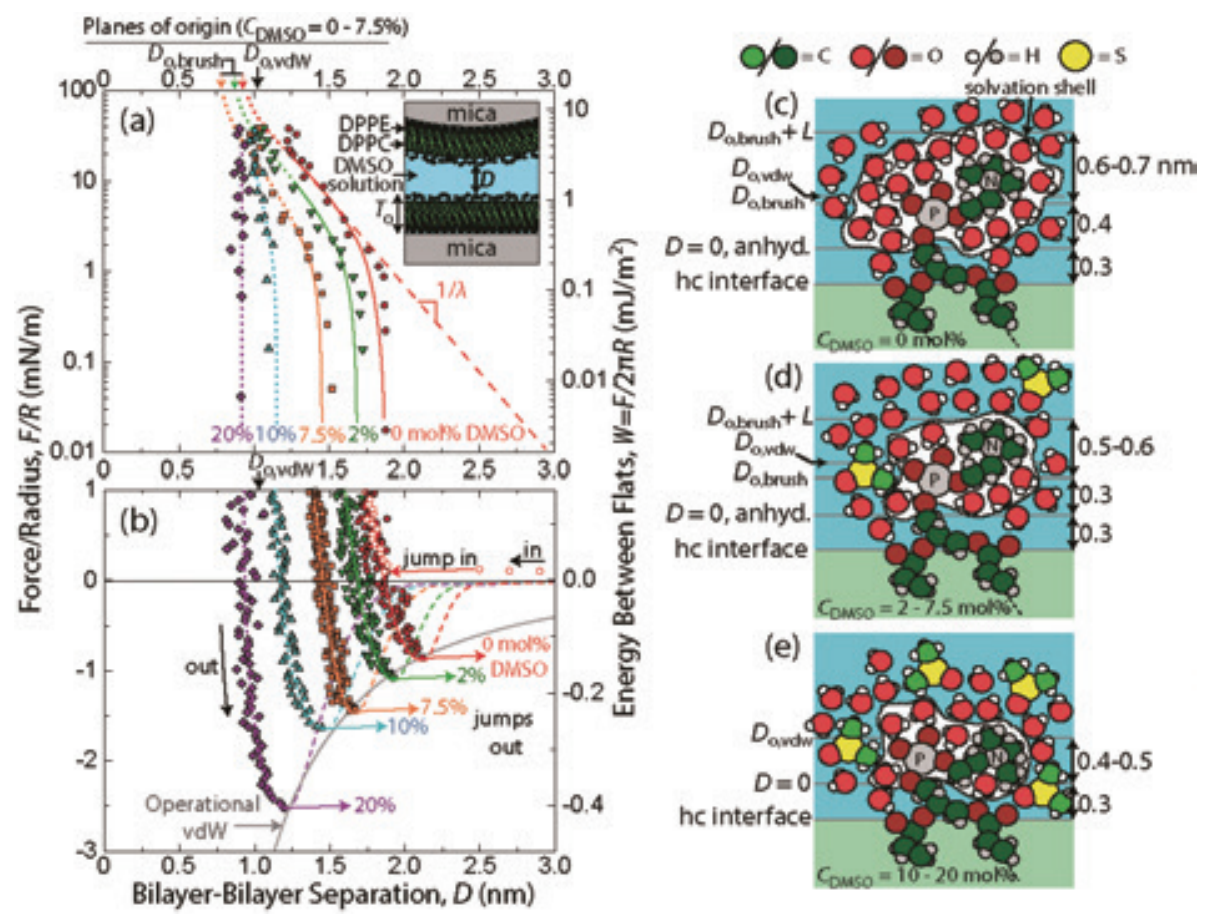

Fig. 6 (a,b) Interaction forces measured between gel-phase DPPC bilayers in DMSO-water mixtures ranging from DMSO concentrations of $0-20 \mathrm{~mol} \%$. Solid lines are fits of polymer brush and vdW forces, whereas $10 \%$ and $20 \%$ DMSO samples were unable to be fitted with the polymer brush equation. (c) Schematic views of the PC headgroups as a function of DMSO concentrations. The $D_{\mathrm{o}, \mathrm{vdw}}, D_{\mathrm{o}, \mathrm{brush}}$, and $L$ indicate the vdW plane of origin, brush plane of origin and brush length, respectively. [Adapted and modified from Schrader et al., 2015 ${ }^{35)}$ ]

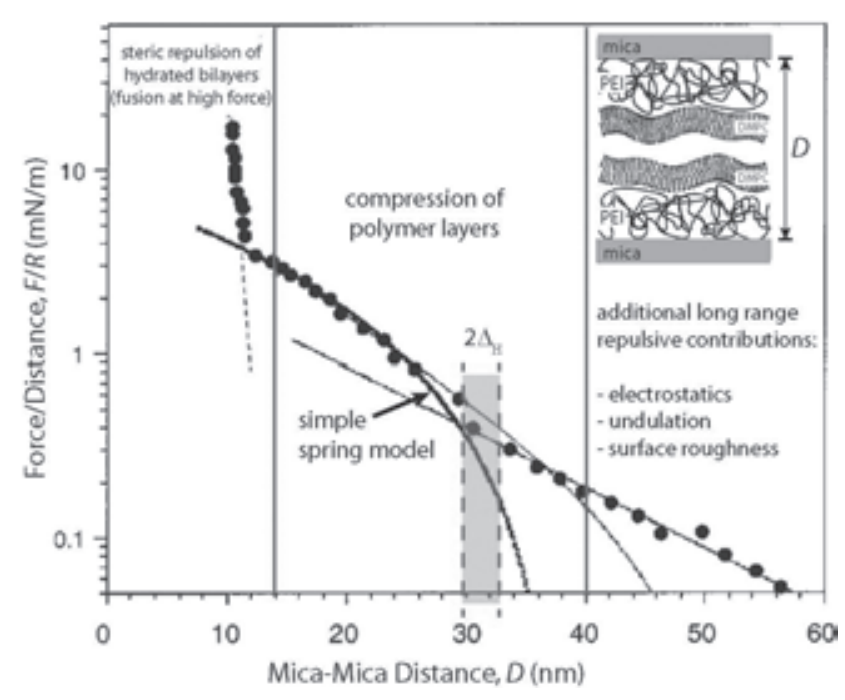

Fig. 7 Interaction profile of two branched polyethylenimine (PEI) - supported DMPC bilayers. $\Delta_{\mathrm{H}}$ indicates hydrodynamic layer thickness. [Adapted and modified from Seitz et al., 2001 ${ }^{38)}$.]

$$
P=\frac{3 \pi^{2}(k T)^{2}}{64 K_{\mathrm{b}} D^{3}}
$$

where $K_{\mathrm{b}}$ is the bending modulus of bilayers and $D$ is the mean distance between bilayers.

The undulation force in only significant when the lipid bilayers are "free" and "unstressed." But in typical SFA experiments, lipid bilayers are supported on mica surfaces that suppress thermal undulation forces. Meanwhile, Seitz et $a l .{ }^{38)}$, deposited bilayers on a "polymer cushion" rather than a solid mica surface, which indeed increased the magnitude of thermal undulation forces (Fig. 7).

\subsection{Attractive hydrophobic interaction and hemifusion of lipid membranes}

The hydrophobic force is an entropically originated attractive force that has been known to be stronger than $\mathrm{vdW}$ forces. Even though the origin, range, decay length, and the full potential function are still not fully understood, there is no doubt that hydrophobic interaction is one of the most important interactions in biological systems. It is mainly responsible for biomolecular interaction, membrane fusion, protein folding, and so on. Specifically, the self-assembly of lipids to lipid bilayers is also highly dominated by hydrophobic interaction between hydrocarbon tails.

When bilayers are free (unstressed), the hydrophilic headgroups shield the hydrophobic interior packed with hydrocarbons, screening the hydrophobic interaction between two bilayers. However, when the bilayers are subjected to tensile stress, normal compression, or depletion, 


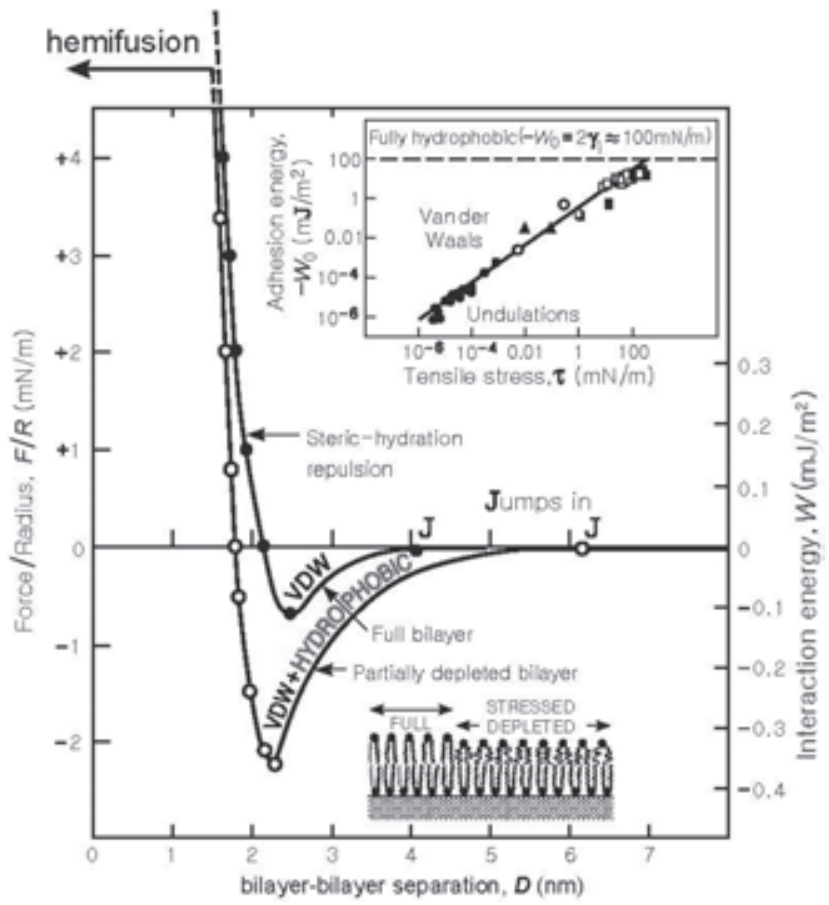

Fig. 8 Interaction forces measured between unstressed and partially stressed bilayers. Hydrocarbon chains are effectively shielded from aqueous phase in unstressed bilayers, whereas those of the stressed bilayers expose additional unshielded hydrophobic groups, which results in additional hydrophobic interaction in addition to $\mathrm{vdW}$ interaction. [Adapted and modified from Israelachvili, 2001 ${ }^{20)}$.]

a fraction of the exposed area of the hydrophobic interior increases compared to the hydrophilic headgroup area. Exposure of the hydrophobic interior enables the bilayers to interact via hydrophobic interaction, which is strong enough to make two lipid bilayers hemifuse upon approach and/or compression(Fig. 8).
Using the SFA, many studies have measured hemifusion of mica- or polymer-supported lipid bilayers as functions of temperature $^{39)}$, lipid phase and composition ${ }^{39-41)}$, calcium ion concentration ${ }^{40,42)}$, and external pressure ${ }^{4)}$. In all cases, the core cause of the hemifusion narrows down to whether the hydrophobic interior is sufficiently exposed to the aqueous environment. Moreover, the initial break point during hemifusion was observed with FECO, and if the bilayers are adhesive and exhibit Johnson-Kendall-Roberts (JKR) contact, then they seem to break from the edges of the lipid bilayers, whereas repulsive bilayers with Hertzian contact seem to fuse from the center where the pressure is the highest (Fig. 9).

The recent work by Donaldson et al. ${ }^{43)}$ developed a general hydrophobic interaction potential for lipid bilayers that accounts for the deformation of the lipid monolayer upon compression. The overall model, which includes the DLVO, steric hydration, and hydrophobic interaction, successfully predicted the hemifusion event of azobenzene trimethylammonium bromide (azoTAB) bilayers (Fig. 10). The equation includes variables and parameters such as hydrophobic decay length $\left(D_{\text {hydro }} \approx 1 \mathrm{~nm}\right)$, distance between bilayers $(d)$, unstressed molecular area $\left(a_{0}\right)$, molecular area $(a)$, and interfacial tension $(\gamma)$ :

$$
E_{\text {hydro }}=-\gamma\left(a-a_{0}\right) e^{-d / D_{\text {Hydro }}}
$$

Very recently, using an SFA that was attached with fluorescence microscopy, interaction forces between lipid bilayers were measured during the membrane hemifusion event with simultaneous fluorescence imaging (Fig. 11). The hemifusion started at the contact where liquid-disordered $\left(\mathrm{L}_{\mathrm{d}}\right)$ phases of bilayers overlapped, which is the weakest point exposing the highest fraction of hydrophobic interior. Also, high compressive pressure accelerated the hemifusion process because of the increased fraction of exposed hydrophobic interior.

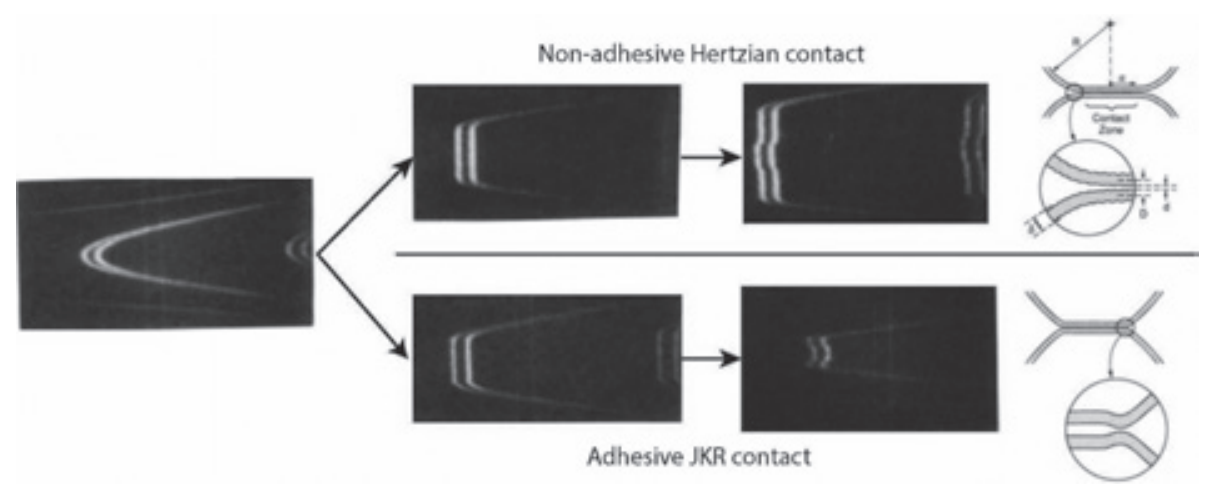

Fig. 9 The optical fringe patterns of two bilayers. The shapes of these fringes at equal chromatic order(FECO) show that there can be two separate scenarios during membrane hemifusion: (i) non-adhesive contact case where hemifusion starts from center of the contact, and (ii) adhesive contact case where hemifusion starts from the edge of the contact. [Adapted and modified from Helm et al., 1992 ${ }^{41)}$.] 


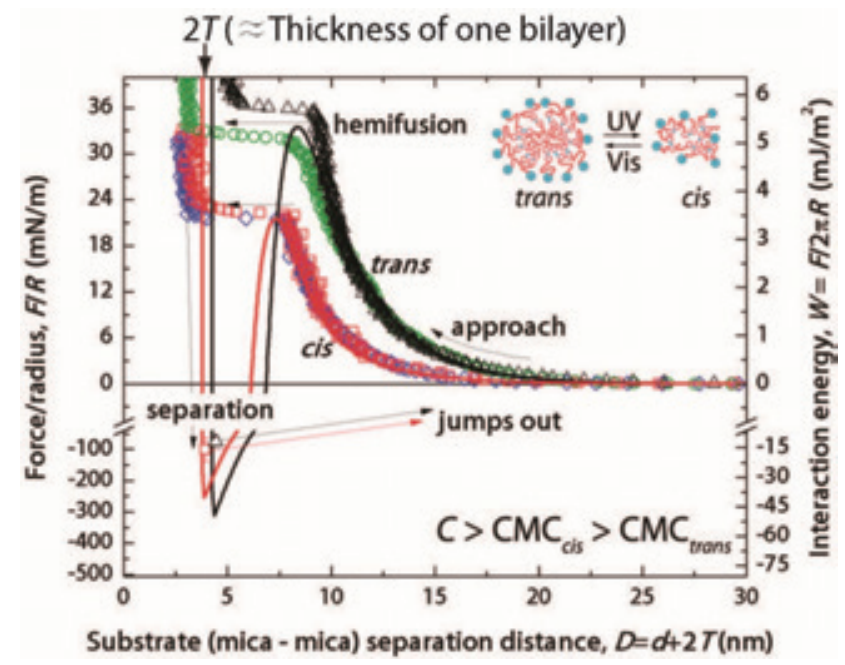

Fig. 10 Interaction forces for adsorbed layers from spontaneous self-assembly of $12 \mathrm{mM}$ azoTAB, which is above the critical micelle concentrations of both the cis and trans azoTAB. [Adapted and modified from Donaldson et al., 2011 ${ }^{43)}$.]

\subsection{Interbilayer interactions mediated by other molecules}

Interbilayer interactions can be mediated by other molecules located between or within bilayers, such as membrane proteins, polymers, and so on. In various SFA experiments, a wide range of molecules was injected between or grafted to the lipid bilayers to investigate how these molecules modulated intermembrane interaction potential.

\subsubsection{Biomolecule bridging}

Membrane proteins can bridge two lipid bilayers generally by electrostatic, vdW, and hydrophobic interactions. One of the most extensively studied membrane proteins using the SFA is myelin basic protein (MBP), which is known to act as a protein glue bridging lipid bilayers together in a tissue called myelin. Various SFA studies have examined the role of $\mathrm{MBP}^{44-46)}$ and lipid composition ${ }^{40,46)}$ on the intermembrane forces, because low intermembrane adhesion may cause disruption of myelin structure leading to a disease known as multiple sclerosis (MS). It was found that with an optimum amount of MBP between bilayers, the intermembrane adhesion is enhanced, which is important for the integrity of myelin structure ${ }^{44-46)}$. Also, lipid compositions are highly responsible for the formation of lipid rafts ${ }^{47,48)}$, which can control the adsorption of MBP onto the bilayers (Fig. 12), where normal (healthy) myelin exhibited higher adhesion and more compact MBP layers compared to those of experimental allergic encephalomyelitis (EAE; an animal model for MS) myelin ${ }^{46)}$.

Lipid bilayers can also be used as templates for measuring interactions between biomolecules. Helm et al. ${ }^{49)}$ measured biotin-streptavidin interaction by using biotin-conjugated lipid molecules. The biotin-conjugated lipids were mixed with normal lipids at a desired ration during LB deposition of lipid bilayers. Streptavidin solution was injected between two biotin-conjugated bilayers to directly measure the ligand-receptor interaction forces, which was found to be a very strong and very short-range ( $<1 \mathrm{~nm}$ ) attractive force (Fig. 13).

2.5.2 Polymer-mediated interactions

Many studies have reported interaction forces between bilayers mediated by poly (ethylene glycol) (PEG), which is reported as a biocompatible polymer. Kuhl et al. ${ }^{50,51)}$ reported that PEG usually does not adsorb to bilayer surfaces and acts as a depletant, resulting in depletion attraction between lipid bilayers. However, the low molecular weight of $\mathrm{PEG}(\mathrm{MW} \sim 1 \mathrm{kDa})$ is too small to generate a noticeable effective depletion layer. Also, high MW PEG ( $\sim 20 \mathrm{kDa})$
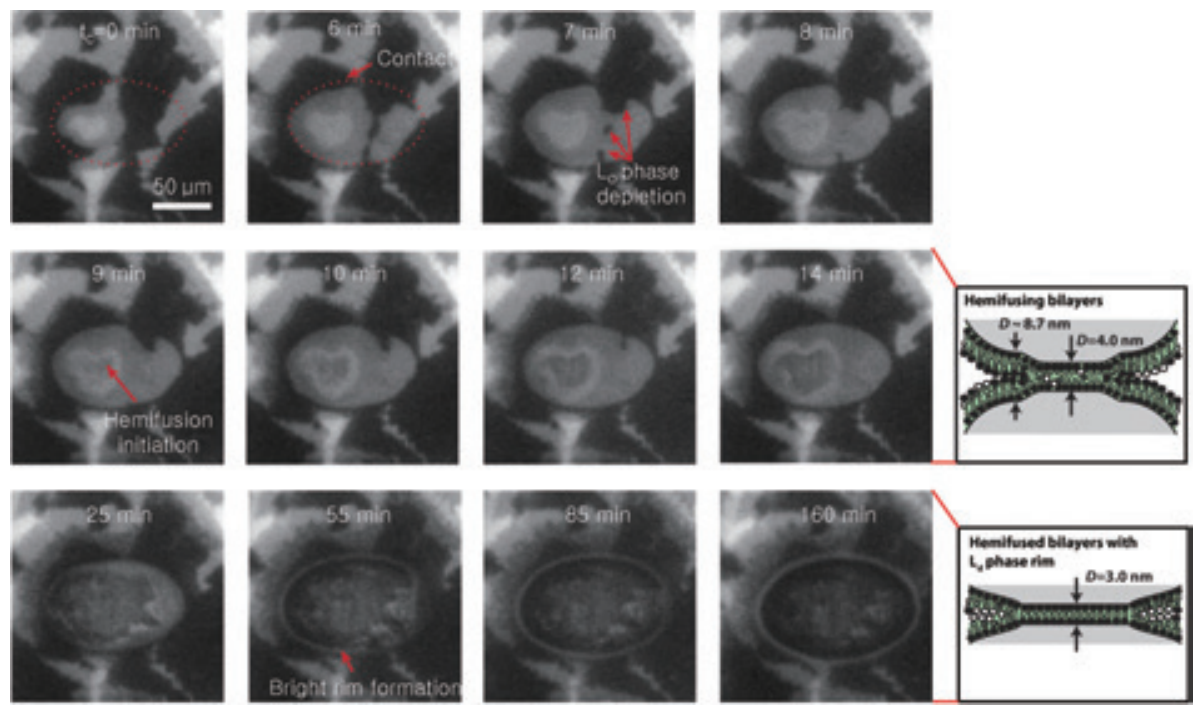

Fig. 11 Evolution of two lipid bilayer contact over time after compression. The black, gray, and white colors indicate $\mathrm{L}_{0}-\mathrm{L}_{\mathrm{o}}$, $\mathrm{L}_{0}-\mathrm{L}_{\mathrm{d}}$ and $\mathrm{L}_{\mathrm{d}}-\mathrm{L}_{\mathrm{d}}$ phase overlaps, respectively. [Adapted and modified from Lee et al., 2015 ${ }^{4)}$.] 


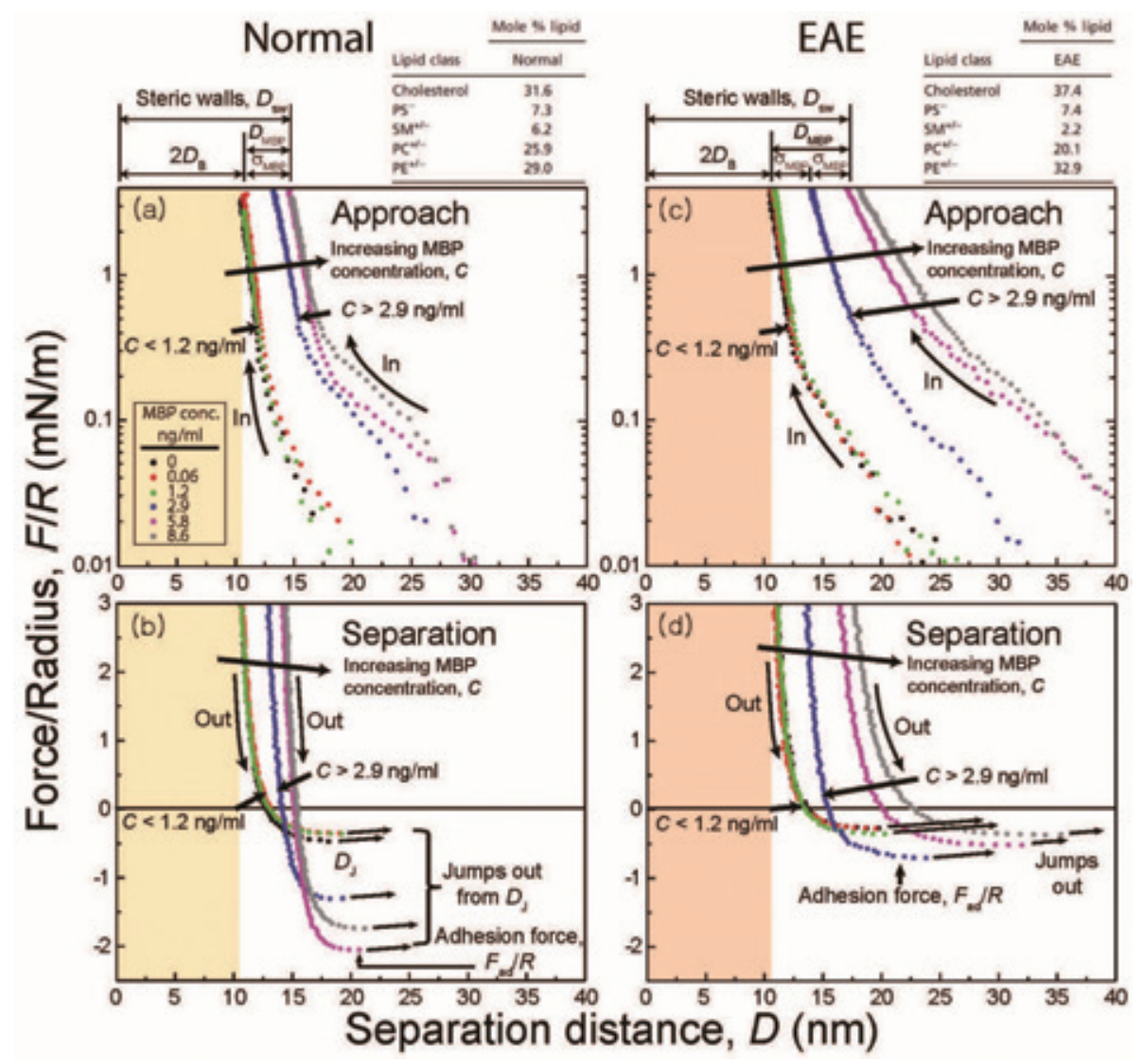

Fig. 12 Interaction forces between myelin lipid bilayers as functions of lipid compositions (normal vs. EAE) and myelin basic protein compositions $(0-8.6 \mathrm{ng} / \mathrm{ml})$. [Adapted and modified from Lee et al., 2014 ${ }^{46)}$.]
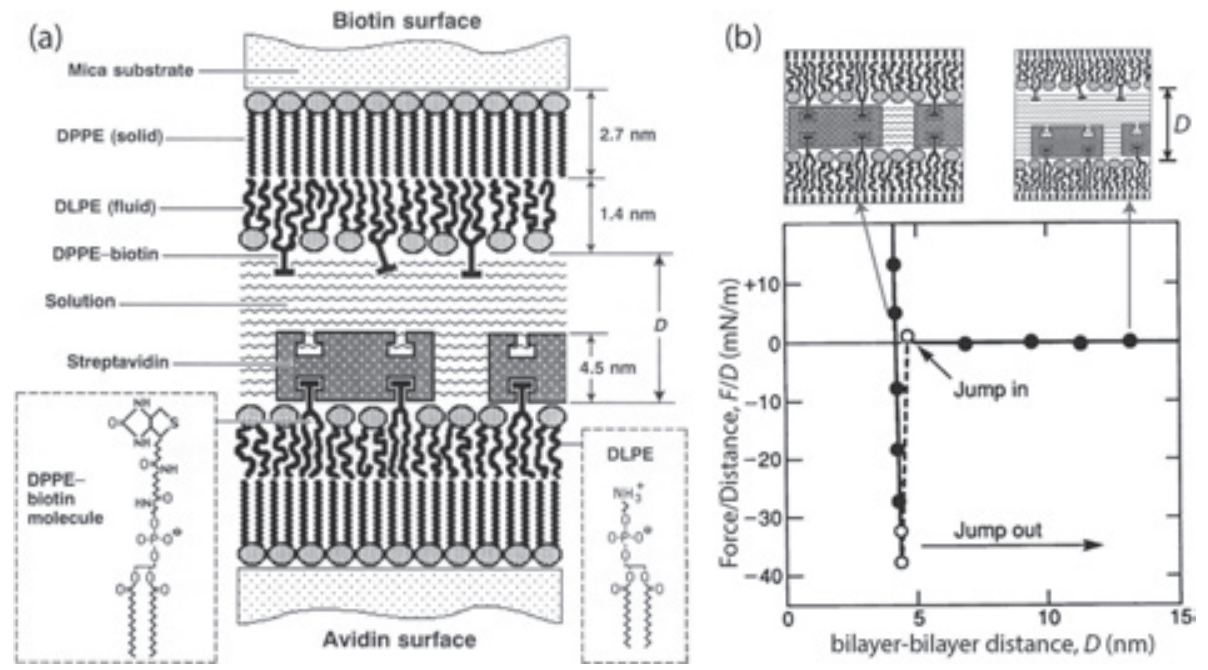

Fig. 13 (a) Schematic of experimental set-up for biotin-streptavidin interaction measurement, and (b) force-distance curve of the biotin-streptavidin interaction. [Adapted and modified from Helm et al., 1991 ${ }^{49)}$.]

adsorbs to bilayer surfaces, resulting in steric repulsion rather than depletion. Thus, proper MW and concentrations of PEG should be used to trigger depletion interaction between bilayers, which can induce fusion.

Rather than using free PEG molecules, Kuhl et al. ${ }^{52)}$ used
PEG-conjugated lipid molecules when making lipid bilayers. The surface density of PEG can be controlled by mixing PEG-conjugated lipids with normal lipids in a desired ratio. At low PEG surface density $(\sim 1.3 \%)$, a nonoverlapping "mushroom" type interaction force was mea- 


\section{W. Lee}
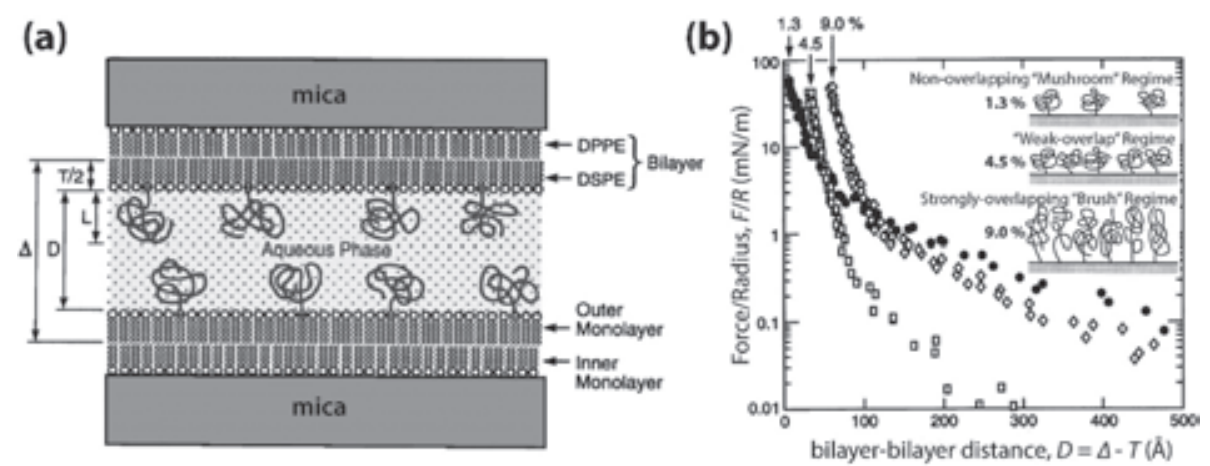

Fig. 14 (a) Schematic of the bilayer-coated mica surfaces with grafted PEG and (b) interaction forces between them as a function of PEG coverage (1.3-9.6 \%). [Adapted and modified from Kuhl et al., $1994^{52)}$.]

sured, whereas at high PEG surface density $(\sim 9.0 \%)$, strongly repulsive "brush" type interaction was observed (Fig. 14).

Banquy et al. ${ }^{53)}$ measured interactions between two lipid bilayers mediated by Pluronic polymers[poly (PEG/poly (propylene oxide) (PPO)/PEG) block copolymer] as a function of polymer concentration. Below the critical aggregate concentration, Pluronics polymers adsorb to lipid bilayers causing the dominant interaction to be steric repulsion. Above the critical aggregate concentration, the onset of interaction forces increases significantly. Moreover, F127, which possesses a larger PPO block compared to F68, exhibits stronger binding to lipid membranes by the partial penetration of the PPO block into the bilayers, which is manifested by a thick gel-like layer formation.

\section{Concluding remarks}

Using an SFA is a powerful technique for measuring the interaction forces and absolute distance between two macroscopic surfaces. Due to its fine distance and force resolutions, the SFA technique was used to resolve different types of forces between lipid bilayers, including non-specific interactions such as vdW, electrostatic DL, steric hydration, thermal undulation, and hydrophobic interactions, as well as specific interactions such as ligand-receptor binding. Even though the technique is excellent for studying fundamentals of intermembrane interactions, mimicry of real cells still remains a challenge due to its (i) solid supported characteristics, (ii) difficulties in mimicking proteinincorporated membrane structures (transmembrane proteins), and (iii) discrepancies in the radius of curvature. However, with clever strategies (e.g., soft polymer cushion below lipid bilayers, high curvature disks, and spontaneous self-assembly of transmembrane protein reconstituted liposome), the SFA technique will be able to measure intermembrane forces that can closely represent the interaction forces of naturally occurring liposomes and cells. Moreover, using the recently developed fluorescence microscope at- tachment, simultaneous imaging of lipid phases and/or other molecules (e.g., membrane proteins) is possible during the approach/retraction cycle of two lipid bilayers. These experiments will unambiguously reveal the cooperative role of lipid phases and membrane proteins during cell/ liposome adhesion and fusion.

\section{Acknowledgement}

This review was supported by a grant from the National Research Foundation of Korea funded by the Korean Government(NRF-2016R1C1B2014294) and by the "Project for Discovering Outstanding Basic Research Ideas" Research Fund (1.180068.01) of UNIST (Ulsan National Institute of Science \& Technology).

\section{References}

1) Tabor, D.; Winterton, R.H.S. Direct measurement of normal and retarded van der waals forces. Proc. $R$. Soc. London, Ser. A 312, 435-450 (1969).

2) Israelachvili, J.N.; Tabor, D. The Measurement of Van Der Waals Dispersion Forces in the Range 1.5 to 130 nm. Proc. R. Soc. London, Ser. A 331, 19-38 (1972).

3) Israelachvili, J.; Min, Y.; Akbulut, M.; Alig, A.; Carver, G.; Greene, W.; Kristiansen, K.; Meyer, E.; Pesika, N.; Rosenberg, K.; Zeng, H. Recent advances in the surface forces apparatus (SFA) technique. Rep. Prog. Phys. 73, 16 (2010).

4) Lee, D.W.; Kristiansen, K.; Donaldson, S.H.; Cadirov, N.; Banquy, X.; Israelachvili, J.N. Real-time intermembrane force measurements and imaging of lipid domain morphology during hemifusion. Nat. Commun. 6, 8(2015).

5) Banquy, X.; Lee, D.W.; Das, S.; Hogan, J.; Israelachvili, J.N. Shear-induced aggregation of mammalian synovial fluid components under boundary lubrication conditions. Adv. Funct. Mater. 24, 3152-3161(2014). 
6) Valtiner, M.; Banquy, X.; Kristiansen, K.; Greene, G.W.; Israelachvili, J.N. The electrochemical surface forces apparatus: The effect of surface roughness, electrostatic surface potentials, and anodic oxide growth on interaction forces, and friction between dissimilar surfaces in aqueous solutions. Langmuir 28, 1308013093 (2012).

7) Israelachvili, J.N.; Kristiansen, K.; Gebbie, M.A.; Lee, D.W.; Donaldson, S.H.; Das, S.; Rapp, M.V.; Banquy, X.; Valtiner, M.; Yu, J. The intersection of interfacial forces and electrochemical reactions. J. Phys. Chem. B 117, 16369-16387 (2013).

8) Israelachvili, J.N. Thin film studies using multiplebeam interferometry. J. Colloid Interface Sci. 44, 259-272 (1973).

9) Shrestha, L.K.; Strzelczyk, K.M.; Shrestha, R.G.; Ichikawa, K.; Aramaki, K.; Hill, J.P.; Ariga, K. Nonionic amphiphile nanoarchitectonics: self-assembly into micelles and lyotropic liquid crystals. Nanotechnology 26, 11 (2015).

10) Denisov, I.G.; Sligari, S.G. Nanodiscs in membrane biochemistry and biophysics. Chem. Rev. 117, 4669-4713 (2017).

11) Abdulreda, M.H.; Moy, V.T. Atomic force microscope studies of the fusion of floating lipid bilayers. Biophys. J. 92, 4369-4378(2007).

12) Zasadzinski, J.A.; Viswanathan, R.; Madsen, L.; Garnaes, J.; Schwartz, D.K. Langmuir-blodgett-films. Science 263, 1726-1733 (1994).

13) Tamm, L.K.; McConnell, H.M. Supported phospholipidbilayers. Biophys. J. 47, 105-113(1985).

14) Brian, A.A.; McConnell, H.M. Allogeneic stimulation of cytotoxic T cells by supported planar membranes. Proc. Natl. Acad. Sci. USA 81, 6159-6163(1984).

15) Kalb, E.; Frey, S.; Tamm, L.K. Formation of supported planar bilayers by fusion of vesicles to supported phospholipid monolayers. Biochim. Biophys. Acta 1103, 307-316 (1992).

16) Pashley, R.M.; Israelachvili, J.N. A comparison of surface forces and interfacial properties of mica in purified surfactant solutions. Colloids Surf. 2, 169-187 (1981).

17) Marra, J.; Israelachvili, J. Direct measurements of forces between phosphatidylcholine and phosphatidylethanolamine bilayers in aqueous-electrolyte solutions. Biochemistry 24, 4608-4618(1985).

18) Marra, J. Direct measurements of attractive Van der Waals and adhesion forces between uncharged lipid bilayers in aqueous solutions. J. Colloid Interface Sci. 109, 11-20 (1986).

19) Marra, J. Direct measurement of the interaction between phosphatidylglycerol bilayers in aqueous electrolyte solutions. Biophys. J. 50, 815-825 (1986).

20) Israelachvili, J.N. Intermolecular and Surface Forc- es, 3rd ed. Academic Press, London, (2011).

21) Verwey, E.J.W.; Overbeek, J.T.G. Theory of the Stability of lyophobic Colloids. Elsevier, Amsterdam (1948).

22) Ninham, B.W.; Parsegian, V.A. Electrostatic potential between surfaces bearing ionizable groups in ionic equilibrium with physiologic saline solution. J. Theor. Biol. 31, 405-428 (1971).

23) Chan, D.Y.C.; Mitchell, D.J.; Ninham, B.W.; Pailthorpe, B.A. Solvent structure in particle interactions. Low pressure effects and analytic limits. J. Chem. Soc., Faraday Trans.2 76, 776-784(1980).

24) Ohshima, H.; Chan, D.Y.C.; Healy, T.W.; White, L.R. Improvement on the Hogg-Healy-Fuerstenau formulas for the interaction of dissimilar double layers: II. Curvature correction to the formula for the interaction of spheres. J. Colloid Interface Sci. 92, 232-242 (1983).

25) Ohshima, H. Electrostatic interaction between two dissimilar spheres: An explicit analytic expression. $J$. Colloid Interface Sci. 162, 487-495 (1994).

26) Ohshima, H. Electrostatic interaction between two dissimilar spheres with constant surface charge density. J. Colloid Interface Sci. 170, 432-439 (1995).

27) Sharp, K.A.; Honig, B. Electrostatic interactions in macromolecules - theory and applications. Annu. Rev. Biophys. Biophys. Chem. 19, 301-332 (1990).

28) Guldbrand, L.; Jonsson, B.; Wennerstrom, H.; Linse, P. Electrical double-layer forces - a monte-carlo study. $J$. Chem. Phys. 80, 2221-2228 (1984).

29) Joensson, B.; Wennerstroem, H.; Halle, B. Ion distributions in lamellar liquid crystals. A comparison between results from Monte Carlo simulations and solutions of the Poisson-Boltzmann equation. J. Phys. Chem. 84, 2179-2185 (1980).

30) Manning, G.S. Limiting laws and counterion condensation in polyelectrolyte solutions .1. colligative properties. J. Chem. Phys. 51, 924 (1969).

31) Langmuir, I. The role of attractive and repulsive forces in the formation of tactoids, thixotropic gels, protein crystals and coacervates. J. Chem. Phys. 6, 873-896 (1938).

32) Israelachvili, J.N.; Wennerstroem, H. Hydration or steric forces between amphiphilic surfaces? Langmuir 6, 873-876 (1990).

33) Israelachvili, J.N.; Wennerstroem, H. Entropic forces between amphiphilic surfaces in liquids. J. Chem. Phys. 96, 520-531 (1992).

34) Israelachvili, J.; Wennerstrom, H. Role of hydration and water structure in biological and colloidal interactions. Nature 379, 219-225 (1996).

35) Schrader, A.M.; Donaldson, S.H.; Song, J.; Cheng, C.Y.; Lee, D.W.; Han, S.; Israelachvili, J.N. Correlating steric hydration forces with water dynamics through surface force and diffusion NMR measurements in a lipid-DM- 


\section{W. Lee}

SO-H2O system. Proc. Natl. Acad. Sci. USA 112, 10708-10713 (2015).

36) Servuss, R.M.; Helfrich, W. Mutual adhesion of lecithin membranes at ultralow tensions. J. Phys. 50, 809-827 (1989).

37) Helfrich, W. Steric interaction of fluid membranes in multilayer systems. Z. Naturfors. Sect. A-J. Phys. Sci. 33, 305-315 (1978).

38) Seitz, M.; Park, C.K.; Wong, J.Y.; Israelachvili, J.N. Long-range interaction forces between polymer-supported lipid bilayer membranes. Langmuir 17, 46164626 (2001).

39) Wong, J.Y.; Park, C.K.; Seitz, M.; Israelachvili, J. Polymer-cushioned bilayers. II. An investigation of interaction forces and fusion using the surface forces apparatus. Biophys. J. 77, 1458-1468(1999).

40) Banquy, X.; Kristiansen, K.; Lee, D.W.; Israelachvili, J.N. Adhesion and hemifusion of cytoplasmic myelin lipid membranes are highly dependent on the lipid composition. Biochim. Biophys. Acta 1818, 402-410 (2012).

41) Helm, C.A.; Israelachvili, J.N.; McGuiggan, P.M. Role of hydrophobic forces in bilayer adhesion and fusion. Biochemistry 31, 1794-1805(1992).

42) Leckband, D.E.; Helm, C.A.; Israelachvili, J. Role of calcium in the adhesion and fusion of bilayers. Biochemistry 32, 1127-1140 (1993).

43) Donaldson, S.H.; Lee, C.T.; Chmelka, B.F.; Israelachvili, J.N. General hydrophobic interaction potential for surfactant/lipid bilayers from direct force measurements between light-modulated bilayers. Proc. Natl. Acad. Sci. USA 108, 15699-15704(2011).

44) Hu, Y.F.; Doudevski, I.; Wood, D.; Moscarello, M.; Husted, C.; Genain, C.; Zasadzinski, J.A.; Israelachvili, J. Synergistic interactions of lipids and myelin basic protein. Proc. Natl. Acad. Sci. USA 101, 13466-13471 (2004).

45) Min, Y.; Kristiansen, K.; Boggs, J.M.; Husted, C.; Zasadzinski, J.A.; Israeiachvill, J. Interaction forces and ad- hesion of supported myelin lipid bilayers modulated by myelin basic protein. Proc. Natl. Acad. Sci. USA 106, 3154-3159 (2009).

46) Lee, D.W.; Banquy, X.; Kristiansen, K.; Kaufman, Y.; Boggs, J.M.; Israelachvili, J.N. Lipid domains control myelin basic protein adsorption and membrane interactions between model myelin lipid bilayers. Proc. Natl. Acad. Sci. USA 111, E768-E775(2014).

47) Sezgin, E.; Levental, I.; Mayor, S.; Eggeling, C. The mystery of membrane organization: composition, regulation and roles of lipid rafts. Nat. Rev. Mol. Cell Biol. 18, 361-374 (2017).

48) Lee, D.W.; Min, Y.J.; Dhar, P.; Ramachandran, A.; Israelachvili, J.N.; Zasadzinski, J.A. Relating domain size distribution to line tension and molecular dipole density in model cytoplasmic myelin lipid monolayers. Proc. Natl. Acad. Sci. USA 108, 9425-9430 (2011).

49) Helm, C.A.; Knoll, W.; Israelachvili, J.N. Measurement of ligand receptor interactions. Proc. Natl. Acad. Sci. USA 88, 8169-8173(1991).

50) Kuhl, T.; Guo, Y.Q.; Alderfer, J.L.; Berman, A.D.; Leckband, D.; Israelachvili, J.; Hui, S.W. Direct measurement of polyethylene glycol induced depletion attraction between lipid bilayers. Langmuir 12, 3003-3014 (1996).

51) Kuhl, T.L.; Berman, A.D.; Hui, S.W.; Israelachvili, J.N. Part 2. Crossover from depletion attraction to adsorption: Polyethylene glycol induced electrostatic repulsion between lipid bilayers. Macromolecules 31, 82588263 (1998).

52) Kuhl, T.L.; Leckband, D.E.; Lasic, D.D.; Israelachvili, J.N. Modulation of interaction forces between bilayers exposing short-chained ethylene-oxide headgroups. Biophys. J. 66, 1479-1488(1994).

53) Banquy, X.; Lee, D.W.; Kristiansen, K.; Gebbie, M.A.; Israelachvili, J.N. Interaction forces between supported lipid bilayers in the presence of PEGylated polymers. Biomacromolecules 17, 88-97(2016). 